\title{
Associação entre fatores socioeconômicos e insegurança alimentar: estudo de base populacional na Região Metropolitana do Rio de Janeiro, Brasil ${ }^{1}$
}

\section{Association between socioeconomic factors and food insecurity: a population-based study in the Rio de Janeiro metropolitan area, Brazil}

\author{
Rosana SALLES-COSTA2 \\ Rosangela Alves PEREIRA ${ }^{2}$ \\ Maurício Teixeira Leite de VASCONCELLOS ${ }^{3}$ \\ Gloria Valeria da VEIGA² \\ Vânia Maria Ramos de MARINS4 \\ Beatriz Cordeiro JARDIM ${ }^{5}$ \\ Fábio da Silva GOMES4,5 \\ Rosely SICHIERI ${ }^{6}$
}

\section{R E S U M O}

\section{Objetivo}

Estimar a prevalência de insegurança alimentar em famílias de Duque de Caxias, município localizado na Região M etropolitana do Rio de Janeiro, e avaliar a associação entre indicadores socioeconômicos e insegurança alimentar.

\footnotetext{
$\overline{1}$ Este estudo faz parte do projeto "Avaliação do estado nutricional, hábitos al imentares e insegurança alimentar no município de Duque de Caxias, Rio de Janeiro: desenvolvimento de um instrumento simplificado para aval iação de consumo alimentar saudável". Apoio financeiro: Ministério da Ciência e Tecnologia, Ministério do Desenvolvimento Social e Combate à Fome e Conselho Nacional de Desenvolvimento Científico e Tecnológico, a partir do Edital MCT/MESA/CN Pq/CT-Agronegócio 01/2003 (processo CNPq no 503139/2003-3), Instituto Nacional do Câncer e Ministério da Saúde.

2 Universidade Federal do Rio de Janeiro, Instituto de Nutrição J osué de Castro, Departamento de Nutrição Social e Aplicada. Av. Carlos Chagas Filho, 373, Edifício do Centro de Ciências da Saúde, Bloco J, 2o andar, Cidade Universitária, 21941-902, Rio de Janeiro, RJ, Brasil. Correspondência para/Correspondence to: R. SALLES-COSTA. E-mail: বrosana_salles@terra.com.br>.

3 Instituto Brasileiro de Geografia e Estatística, Escola N acional de Ciências Estatísticas. Rio de Janeiro, RJ, Brasil.

4 Universidade Federal Fluminense, Faculdade Arthur Sá Earp Neto, Curso de N utrição. Petrópolis, RJ, Brasil.

5 Ministério da Saúde, Instituto Nacional do Câncer, Coordenação de Prevenção e Vigilância, Área de Alimentação, Nutrição e Câncer. Rio de Janeiro, RJ, Brasil.

6 Universidade do Estado do Rio de Janeiro, Instituto de Medicina Social, Departamento de Epidemiologia, Núcleo de Epidemiologia e Biologia da Nutrição. Rio de Janeiro. RJ. Brasil.
} 


\section{Métodos}

Desenvolveu-se estudo transversal, de base populacional, em famílias do distrito de Campos Elíseos, município de Duque de Caxias, Rio de Janeiro, que investigou amostra probabilística composta por 1085 domicílios. As informações sobre condições socioeconômicas foram obtidas por meio de entrevista, utilizando questionário estruturado. A insegurança alimentar foi avaliada com o uso da Escala Brasileira de Insegurança Alimentar, que permite classificar as famílias em segurança alimentar ou em insegurança alimentar leve, moderada ou grave. As análises foram desenvolvidas levando em consideração o efeito do desenho da amostra. Foi estimada a prevalência de insegurança alimentar, avaliando-se sua associação com as variáveis socioeconômicas aplicando-se $o$ teste do qui-quadrado $(p<0,05)$.

\section{Resultados}

A prevalência de insegurança alimentar foi de 53,8\%. As variáveis renda familiar mensal per capita, escolaridade do chefe da família, nível socioeconômico (avaliado segundo os critérios da Associação Brasileira de Institutos de Pesquisa de Mercados), número de pessoas e presença de filtro de água no domicílio, apresentaram associação inversa significante com a insegurança alimentar.

\section{Conclusão}

A renda familiar foi o indicador que discriminou tanto a segurança quanto a insegurança alimentar.

Termos de indexação: Estudos transversais. Fatores socioeconômicos. Insegurança alimentar.

\section{A B S T R A C T}

\section{Objective}

This work aims to evaluate the prevalence of food insecurity among families from Duque de Caxias, in the Rio de Janeiro Metropolitan Area and the association between socioeconomic indicators and food insecurity.

\section{Methods}

A population-based cross-sectional study investigated a probabilistic sample composed of 1,085 households from the district of Campos Elíseos, in the municipality of Duque de Caxias, Rio de Janeiro, Brazil. Information on socioeconomic condition was obtained using a structured questionnaire. Food insecurity was assessed by the Brazilian Food Insecurity Scale, which allows classifying the families into food security, or mild, moderate or severe food insecurity. The analyses took into account the sampling design effect. The food insecurity prevalence was estimated and its association with socioeconomic variables was assessed using the chi-square test $(p<0.05)$.

\section{Results}

Food insecurity prevalence was $53.8 \%$. The following variables were inversely and significantly associated with food insecurity: family monthly per capita income, head of family educational level, socioeconomic level (classified according to the Brazilian Association of Market Research criteria), number of family members, and having a water filter in the household.

\section{Conclusion}

Family income was the variable that discriminated both food security and insecurity.

Indexing terms: Cross-sectional studies. Socioeconomic factors. Food insecurity.

\section{N T R O D U Ç Ã O}

A escala proposta por Radimer et al. ${ }^{1}$ vem sendo aplicada em diversos países para dimensionar a magnitude da insegurança alimentar ${ }^{2-5}$. No Brasil, recentemente, foi validado um instrumento baseado nessa escala, que possibilita o diagnóstico de forma prática e rápida da situação de segurança alimentar familiar ${ }^{6}$. Essa escala tem sido reconhecida como indicador sensível para detectar famílias em risco de insegurança alimentar?.

A insegurança alimentar é determinada, principalmente, pela pobreza e pelas desigualdades sociais ${ }^{8}$. Estudos que analisam fatores associados à insegurança alimentar são decisivos para o planejamento de programas e políticas 
públicas de caráter preventivo e promoção da saúde9.

As repercussões da insegurança alimentar podem ser observadas, principalmente, nos grupos mais vulneráveis. A mortalidade infantil, o prejuízo do desenvolvimento físico e mental, o baixo peso ao nascer, a mortalidade materna, o aumento da evasão escolar e a diminuição do desempenho acadêmico são eventos relacionados à carência de alimentação saudável e de qualidade, como conseqüência do acesso precário a renda e a bens e serviços ${ }^{10}$.

O município de Duque de Caxias, localizado na Região M etropolitana do Rio de Janeiro, concentra a maior parte das indústrias e dos serviços especializados do Estado, sendo suas principais atividades econômicas a indústria de transformação, a prestação de serviço e o comércio $^{11}$. Entretanto, $14,5 \%$ da população do município auferem rendimentos abaixo da linha de pobreza extrema, ou seja, menos que um dólar americano por dia ${ }^{12}$, proporção que corresponde a quase o dobro da média do estado do Rio de Janeiro $(8,7 \%)^{13}$. Este trabalho tem como objetivos estimar a prevalência de insegurança alimentar entre as famílias do distrito de Campos Elíseos, considerado um dos mais pobres do município, e avaliar a sua associação com indicadores socioeconômicos.

\section{M É T O D O S}

Trata-se de um estudo transversal, de base populacional, que investigou crianças (6 a 30 meses), adolescentes (12 a 18 anos) e adultos residentes em Campos Elíseos, segundo distrito do município de Duque de Caxias, localizado na Região Metropolitana do Rio de Janeiro.

Para tanto, foi utilizada uma amostra probabilística de 1125 domicílios particulares permanentes (DPP) de Campos Elíseos, selecionada em três estágios (setor censitário, domicílio e o indivíduo). 0 tamanho da amostra foi fixado com base em uma estimativa de $14,5 \%$ para a prevalência de pobreza extrema, fixando-se um erro relativo máximo de 5,0\%. No primeiro estágio, dentre os 322 setores constantes da Base Operacional Geográfica (BOG) de 2000 do Instituto Brasileiro de Geografia e Estatística ${ }^{14}$ foram selecionados, de forma sistemática, 75 setores censitários, com probabilidades proporcionais ao número de DPP observados pelo Censo Demográfico de 2000. Previamente à seleção, os setores censitários foram ordenados por renda média do responsável pelo domicílio, de forma a assegurar a representação de todos os níveis de renda na amostra. De fato, a combinação desse método de seleção com a ordenação por renda corresponde a uma estratificação implícita dos setores por renda.

Entre outubro de 2004 e janeiro de 2005, antes da seleção dos domicílios, foi realizada, uma varredura completa dos DPP dos 75 setores censitários selecionados para localizar os endereços e identificar os DPP com crianças e adolescentes. Com esse levantamento foi possível estratificar osDPP de cada setor nos seguintes quatro estratos: (a) DPP só com adultos; (b) DPP com adultos e adolescentes; (c) DPP com adultos e crianças; e (d) DPP com adultos, adolescentes e crianças. Definiu-se um tamanho amostral de 15 DPP por setor. Os DPP foram selecionados com eqüiprobabilidade e alocados de forma a assegurar um mínimo de dois DPP por estrato em cada setor, além de um tamanho amostral que permitisse estimar os parâmetros desejados para crianças e adolescentes. No terceiro estágio, em cada DPP foi selecionado aleatoriamente um morador de cada grupo (criança, adolescente ou adulto), observando-se que no estrato de DPP só com adultos foram selecionados dois adultos em cada DPP selecionado.

A coleta de dados foi realizada no período de maio a dezembro de 2005 . Todos os procedimentos foram realizados por equipe treinada. Foi realizada dupla digitação dos dados no programa CSPRO versão $3.2^{15}$. O tamanho final da amostra foi de 1085 domicílios, com 3,4\% de não resposta. 


\section{Avaliação de insegurança alimentar e nutricional}

A Escala Brasileira de Insegurança Alimentar (EBIA), proposta e validada para o Brasil por Segall-Corrêa et al. ${ }^{16}$, classifica as famílias em quatro categorias: (a) segurança alimentar; (b) insegurança alimentar leve (IA leve); (c) insegurança alimentar moderada (IA moderada) e (d) insegurança alimentar grave (IA grave). Essa escala é elaborada a partir de questionário com 15 perguntas referentes à experiência nos últimos três meses de insuficiência alimentar em diversos níveis de intensidade. Inclui desde a preocupação de que a comida possa acabar até a vivência de passar um dia todo sem comer. Cada resposta afirmativa do questionário corresponde a um ponto, portanto, a soma de todas as repostas varia de 0 a 15 pontos. Quando não há nenhuma resposta positiva, a família é classificada em situação de segurança alimentar e a classificação da insegurança alimentar em diferentes gradientes corresponde a patamares diferenciados da soma dos pontos obtidos no questionário ${ }^{17}$.

\section{Avaliação dos indicadores socioeconômicos e demográficos}

Para a avaliação das características dos domicílios foram selecionadas as seguintes variáveis: (a) condições de saneamento básico (tratamento do esgoto, coleta de lixo, abastecimento de água e tratamento doméstico da água); (b) renda familiar mensal per capita (total de rendimentos da família dividido pelo número de moradores, expressa em múltiplos do saláriomínimo, que à época do estudo era de 300 reais); (c) número de pessoas por domicílio; (d) escolaridade do chefe da família (categorizada em: analfabeto, ensino fundamental incompleto, ensino fundamental completo, ensino médio completo e curso universitário completo) e (e) classificação socioeconômica pelo critério da Associação Brasileira de Institutos de Pesquisa de Mercados (ABIPEME), que considera a presença de bens e serviços do domicílio e a escolaridade do chefe da família ${ }^{18}$.

\section{Análise de dados}

Os parâmetros e os respectivos intervalos de confiança de 95\% (IC 95\% ) foram estimados para os dados expandidos considerando o efeito do desenho amostral. Estimou-se a prevalência de insegurança alimentar e realizou-se a caracterização da população estudada segundo as variáveis socioeconômicas. Utilizou-se o teste qui-quadrado com correção de segunda ordem de Rao-Scott ${ }^{19}$ para verificar a associação entre os fatores socioeconômicos e os diferentes graus de IA, considerando significantes quando $p<0,05$.

As Razões de Prevalência (RP) e os seus respectivos intervalos de confiança com nível de 95\% (IC 95\%) foram estimados comparando as categorias extremas das variáveis socioeconômicas e de saneamento que se mostraram associadas à segurança alimentar, com o auxílio do programa $R$ versão $2.5 .0^{20}$, utilizando a biblioteca survey (versão 3.6-8) e o método de Linearização de Taylor $^{21}$. Para os casos em que o denominador da RP era nulo, a análise se restringiu à comparação descritiva das duas prevalências, sem as relacionar matematicamente por meio da RP, já que não existe solução para uma razão com denominador igual a zero.

Todos os demais procedimentos de análise incorporaram fatores de expansão calibrados e informações do plano amostral, e foram realizados no programa Stata versão $9.0^{22}$.

O projeto de pesquisa foi aprovado pelo Comitê de Ética e Pesquisa da Universidade do Estado do Rio de Janeiro (registro CEP/IMS no 02/2004). No momento da entrevista domiciliar, foi apresentado o termo de consentimento, no qual o entrevistado assentava a concordância em participar da pesquisa, após esclarecimentos quanto aos procedimentos a serem empregados, a garantia de sigilo das informações prestadas e a possibilidade de se recusar a participar da investigação. 


\section{RESULTA D O S}

A aplicação da Escala Brasileira de Insegurança Alimentar (EBIA) revelou que, no distrito de Campos Elíseos (Município de Duque de Caxias, RJ), 53,8\% dos domicílios sofriam com a insegurança alimentar em diferentes graus: $31,4 \%$ referiram insegurança alimentar leve, 16,1\%, insegurança alimentar moderada e 6,3\%, insegurança alimentar grave. 0 analfabetismo foi observado em 6,2\% dos chefes das famílias e outros $52,3 \%$ não lograram concluir o ensino fundamental. Por outro lado, o ensino universitário foi registrado para $1,3 \%$ dos chefes de família. Nesse distrito, somente $26,8 \%$ das famílias auferiam renda per capita acima de um saláriomínimo. Considerando a classificação de nível socioeconômico da ABIPEM E, 83,4\% dasfamílias foram classificadas nas classes C e D. Aproximadamente $56,0 \%$ dos domicílios tinham até quatro moradores e cerca de $4,0 \%$ mais de oito moradores. A presença de filtro para tratamento de água foi relatada em $63,6 \%$ das residências (Tabela 1).

Dentre as famílias com renda mensal per capita menor que $1 / 4$ do salário-mínimo, somente $12,0 \%$ referiram segurança alimentar; enquanto que $21,3 \%$ relataram insegurança alimentar grave. À medida que a renda familiar aumentou, a proporção de famílias em segurança alimentar também aumentou, chegando a $76,9 \%$ entre as que tinham renda per capita de um salário-mínimo ou mais. Por outro lado, a proporção de famílias em insegurança alimentar grave reduziu-se com o incremento da renda (Tabela 2).

A relação entre a escolaridade do chefe da família e a percepção de segurança alimentar foi marcadamente mais evidente para a insegurança alimentar grave, que teve prevalência de 7,3\% entre as famílias cujos chefes eram analfabetos ou não haviam completado o ensino fundamental, não sendo observada entre as famílias cujos chefes tinham curso universitário (Tabela 2).
Tabela 1. Caracterização dos domicílios investigados quanto à situação de segurança alimentar, às condições socioeconômicas e de saneamento. Campos Elíseos, Duque de Caxias (RJ), 2005.

\begin{tabular}{|c|c|c|c|}
\hline Características dos domicílios & $\mathrm{n}$ & $\%$ & IC95\% \\
\hline \multicolumn{4}{|c|}{ Situação de segurança alimentar 1085} \\
\hline Segurança alimentar & & 46,2 & $41,1-51,2$ \\
\hline Insegurança alimentar leve & & 31,4 & $26,7-36,1$ \\
\hline Insegurança alimentar moderada & & 16,1 & $12,6-19,5$ \\
\hline Insegurança alimentar grave & & 6,3 & $3,9-8,4$ \\
\hline \multicolumn{4}{|c|}{ Renda familiar mensal per capita ${ }^{1} 1065$} \\
\hline$<1 / 4$ salário-mínimo & & 13,7 & $10,9-16,5$ \\
\hline 1/4 - 1/2 salário-mínimo & & 26,7 & $22,7-30,6$ \\
\hline 1⁄2 - 1 salários-mínimo & & 32,8 & $28,6-36,9$ \\
\hline 1 ou + salários-mínimos & & 26,8 & $22,5-31,1$ \\
\hline $\begin{array}{l}\text { Grau de escolaridade do chefe } \\
\text { da família (anos de estudo) }\end{array}$ & $891^{1}$ & & \\
\hline Analfabeto & & 6,2 & $3,9-8,6$ \\
\hline $\begin{array}{l}\text { Ensino fundamental incompleto } \\
\text { ( }<8 \text { anos) }\end{array}$ & & 52,3 & $47,4-57,1$ \\
\hline $\begin{array}{l}\text { Ensino fundamental completo } \\
\text { (8-10 anos) }\end{array}$ & & 19,1 & $15,3-22,9$ \\
\hline $\begin{array}{l}\text { Ensino médio completo } \\
\text { (11-14 anos) }\end{array}$ & & 20,9 & $17,0-24,8$ \\
\hline $\begin{array}{l}\text { Curso universitário completo } \\
\text { ( } \geq 15 \text { anos) }\end{array}$ & & 1,3 & $0,1-2,6$ \\
\hline Nível socioeconômico (ABIPEME) & $888^{2}$ & & \\
\hline$A+B$ (mais alto) & & 6,3 & $3,5-9,0$ \\
\hline C & & 44,6 & $38,5-50,7$ \\
\hline D & & 38,8 & $32,9-44,8$ \\
\hline $\mathrm{E}$ (mais baixo) & & 10,3 & $6,8-13,3$ \\
\hline $\begin{array}{l}\text { Número de moradores no } \\
\text { domicílio }\end{array}$ & 1085 & & \\
\hline $1-4$ & & 55,9 & $51,0-60,9$ \\
\hline $5-8$ & & 40,2 & $35,2-45,1$ \\
\hline$\geq 8$ & & 3,9 & $2,1-5,5$ \\
\hline Saneamento & 1085 & & \\
\hline Serviço público de coleta de lixo & & 89,5 & $84,9-94,1$ \\
\hline $\begin{array}{l}\text { Rede pública de abastecimento } \\
\text { de água }\end{array}$ & & 64,3 & $56,1-72,5$ \\
\hline Serviço de esgoto & & 80,4 & $73,9-86,8$ \\
\hline $\begin{array}{l}\text { Presença de filtro para tratamento } \\
\text { de água no domicílio }\end{array}$ & & 63,6 & $58,5-68,6$ \\
\hline
\end{tabular}

$\mathrm{IC}=$ intervalo de confiança; ${ }^{1}$ Os questionários foram respondidos por um adulto selecionado aleatoriamente no domicílio; os dados de escolaridade do chefe da família foram obtidos para $82,1 \%$ do número de domicílios; ${ }^{2}$ Para a categorização de acordo com os critérios da Associação Brasileira de Institutos de Pesquisa de Mercados (ABIPEM E), utilizam-se informações sobre a escolaridade do chefe da família, além da presença de bens e serviços no domićlio; como os dados de escolaridade do chefe da família foram obtidos para um número limitado de domicílios, a categorização segundo a ABIPEME só pôde ser desenvolvida para $81,8 \%$ dos domicílios. 
Tabela 2. Situação de segurança alimentar classificada segundo a Escala Brasileira de Segurança Alimentar, de acordo com as características socioeconômicas e de saneamento dos domicílios investigados. Campos Elíseos, Duque de Caxias (RJ), 2005.

\begin{tabular}{|c|c|c|c|c|c|}
\hline & $\begin{array}{l}\text { Segurança } \\
\text { alimentar }\end{array}$ & $\mathrm{IA}^{1}$ Leve & $\mathrm{IA}^{1}$ M oderada & $\mathrm{IA}^{1}$ Grave & $p$-valor $\left(\chi^{2}\right)$ \\
\hline Renda familiar mensal per capita ${ }^{2}$ & & & & & $<0,0001$ \\
\hline$<1 / 4$ salário-mínimo & 12,0 & 41,5 & 25,2 & 21,3 & \\
\hline 1/4 - 1/2 salário-mínimo & 27,0 & 37,8 & 27,8 & 7,4 & \\
\hline 1/2 - 1 salário-mínimo & 51,9 & 33,4 & 10,8 & 3,9 & \\
\hline 1 ou mais salários-mínimos & 76,9 & 18,1 & 5,0 & 0,0 & \\
\hline Escolaridade do chefe da família & & & & & 0,004 \\
\hline Analfabeto e ensino fundamental incompleto & 40,1 & 31,9 & 20,7 & 7,3 & \\
\hline Fundamental completo & 55,0 & 28,6 & 10,2 & 6,2 & \\
\hline Ensino médio completo & 59,6 & 32,6 & 7,1 & 0,7 & \\
\hline Curso universitário & 71,8 & 28,2 & 0 & 0 & \\
\hline Nível socioeconômico (ABIPEME) & & & & & $<0,0001$ \\
\hline$A+B$ (mais alto) & 69,8 & 29,8 & 0,4 & 0,0 & \\
\hline $\mathrm{C}$ & 63,2 & 26,5 & 8,9 & 1,4 & \\
\hline $\mathrm{D}$ & 28,9 & 38,9 & 23,3 & 8,9 & \\
\hline $\mathrm{E}$ (mais baixo) & 31,2 & 24,2 & 28,4 & 16,2 & \\
\hline Número de moradores no domicílio & & & & & $<0,0001$ \\
\hline $1-4$ & 52,9 & 27,31 & 15,2 & 4,6 & \\
\hline $5-8$ & 29,0 & 42,7 & 18,5 & 9,8 & \\
\hline$\geq 8$ & 17,8 & 34,1 & 19,7 & 28,4 & \\
\hline Coleta de lixo & & & & & 0,23 \\
\hline Serviço público & 45,8 & 32,2 & 16,3 & 5,7 & \\
\hline Outro & 49,3 & 25,1 & 15,0 & 10,6 & \\
\hline Abastecimento de água & & & & & 0,85 \\
\hline Rede pública & 47,5 & 30,8 & 15,4 & 6,3 & \\
\hline Outro & 44,0 & 32,5 & 17,5 & 6,2 & \\
\hline Esgoto de dejetos & & & & & 0,22 \\
\hline Serviço de esgoto & 47,6 & 32,7 & 15,3 & 5,3 & \\
\hline Outro & 40,3 & 30,5 & 19,6 & 9,6 & \\
\hline Presença de filtro para tratamento de água & & & & & 0,003 \\
\hline Sim & 51,3 & 28,7 & 15,6 & 4,4 & \\
\hline Não & 36,4 & 36,9 & 17,3 & 9,4 & \\
\hline
\end{tabular}

${ }^{1}$ IA: insegurança alimentar; ${ }^{2}$ Considerando o valor do salário-mínimo no ano de $2005(R \$ 300,00)$.

ABIPEME: Associação Brasileira de Institutos de Pesquisa de Mercados.

O mesmo foi observado para a categorização das famílias segundo os critérios da ABIPEM E: a insegurança alimentar grave foi observada em $16,2 \%$ das famílias da categoria E e não foi relatada entre as famílias da categoria $A$ e B (Tabela 2).

O tamanho da família também se associou à percepção de segurança alimentar: entre as famílias com até quatro membros, a prevalência de insegurança moderada foi de $15,2 \%$ e a de insegurança grave foi de $4,6 \%$. Essas prevalências aumentaram com o aumento do tamanho das famílias, chegando a $28,4 \%$ de insegurança grave e $19,7 \%$ de insegurança moderada entre as famílias com oito ou mais membros (Tabela 2).

As condições de saneamento não apresentaram associação com a percepção de insegurança alimentar; entretanto, a presença de filtro no domicílio associou-se com a insegurança alimentar $(p=0,003)$ : $51,3 \%$ das famílias que relataram ter filtro referiram segurança alimentar e 4,4\%, segurança alimentar grave. Entre as que 
não tinham filtro, 36,4\% relataram segurança alimentar e 9,4\%, insegurança alimentar grave (Tabela 2).

A estimativa das razões de prevalências (RP), comparando as categorias extremas das variáveis socioeconômicas e de saneamento, que se mostraram associados à segurança alimentar, revelou que a renda familiar mensal per capita foi o indicador que melhor discrimina as famílias em risco de insegurança alimentar grave: a probabilidade de encontrar famílias nessa situação entre as que auferiam menos de $1 / 4$ do saláriomínimo per capita foi 21,3 vezes mais elevada do que entre as que tinham renda per capita de, pelo menos, um salário-mínimo. A escolaridade do chefe da família e a categorização pelos critérios da ABIPEM E também permitiram identificar o risco de insegurança alimentar grave. Contudo, o grau de escolaridade do chefe da família não se mostrou um forte preditor de segurança alimentar: a razão de prevalência comparando chefes com grau universitário e aqueles que eram analfabetos ou não tinham completado os oito anos de ensino fundamental foi de 1,7 (Tabela 3).

\section{I S C U S S Ã O}

Este estudo apresenta resultados de investigação de base populacional desenvolvida no distrito de Campos Elíseos, município de Duque de Caxias, Região M etropolitana do Rio de Janeiro, abordando segurança alimentar e condições socioeconômicas. Esse município caracteriza-se por extremos contrastes econômicos, sua arrecadação corresponde à segunda mais elevada do estado, pois sedia indústrias de grande porte, entretanto, 0 índice de pobreza extrema é também um dos mais elevados do estado ${ }^{13}$.

A segurança alimentar foi mensurada com base em questionário simplificado, que dimensiona a percepção de segurança alimentar dada por um informante qualificado. Esse instrumento foi adaptado para o Brasil a partir de uma escala adotada pelo Departamento de Agricultura dos Estados Unidos ${ }^{23}$. Para sua validação no País foram desenvolvidos estudos qualitativos e quantitativos nas cinco macro-regiões ${ }^{5,6}$. A Escala Brasileira de Insegurança Alimentar foi considerada pelo IBGE um instrumento "adequado para elaborar diagnós-

Tabela 3. Razão de prevalência e respectivos intervalos de confiança de 95\% (IC 95\%) para segurança alimentar e insegurança alimentar grave, comparando as categorias extremas das variáveis socioeconômicas e de saneamento. Campos Elíseos, Duque de Caxias (RJ), 2005.

\begin{tabular}{|c|c|c|c|c|c|}
\hline \multirow{2}{*}{ Variáveis socioeconômicas } & \multicolumn{2}{|c|}{ Segurança alimentar } & \multicolumn{3}{|c|}{ Insegurança alimentar grave } \\
\hline & $\mathrm{RP}$ & (IC 95\%) & $\mathrm{RP}$ & & (IC 95\%) \\
\hline \multicolumn{6}{|l|}{ Renda familiar mensal per capita } \\
\hline $\begin{array}{l}1 \text { ou mais salários-mínimos /<1/4 salário-mínimo } \\
<1 / 4 \text { salário-mínimo/1 ou mais salários-mínimos }\end{array}$ & \multicolumn{4}{|c|}{$<1 / 4$ salário-mínimo/1 ou mais salários-mínimos } & \\
\hline \multicolumn{6}{|l|}{ Escolaridade } \\
\hline \multicolumn{6}{|l|}{$\begin{array}{l}\text { Analfabeto e ensino fundamental incompleto /universitário } \\
\text { Nível socioeconômico (ABIPEME) }\end{array}$} \\
\hline$A+B / E$ & 2,20 & $(1,14-3,26)$ & & $\mathrm{NE}^{1}$ & \\
\hline \multicolumn{6}{|l|}{$E / A+B$} \\
\hline \multicolumn{6}{|l|}{ Número de moradores no domicílio } \\
\hline $1-4 />8$ & 2,96 & $(0,44-5,47)$ & & & \\
\hline$>8 / 1-4$ & & & 6,18 & & $(1,31-11,06)$ \\
\hline \multicolumn{6}{|l|}{ Presença de filtro para tratamento da água no domicílio } \\
\hline Sim/Não & 1,41 & $(1,03-1,79)$ & & & \\
\hline Não/Sim & & & 2,09 & & $(0,84-3,35)$ \\
\hline
\end{tabular}

${ }^{1} \mathrm{NE}$ : 0 resultado desta RP não pôde ser estimado, pois o denominador é igual a zero.

RP: Razão de prevalência; ABIPEME: Associação Brasileira de Institutos de Pesquisa de Mercados. 
tico da condição de segurança alimentar e indicar populações sob maior risco de insegurança" ${ }^{7}$.

A Pesquisa Nacional por Amostragem de Domicílios (PNAD) ${ }^{7}$, realizada em 2004, em todo o território nacional, identificou insegurança alimentar em 34,8\% dos domicílios particulares do País, e a prevalência de insegurança alimentar grave era de $6,5 \%$. As condições mais precárias foram observadas na região Nordeste, onde a prevalência global de insegurança alimentar foi de $53,6 \%$, e a de insegurança grave, de $12,4 \%$. $\mathrm{Na}$ Região Sudeste, 30,5\% das famílias viviam em insegurança alimentar, sendo $4,1 \%$, em situação grave. No caso particular do estado do Rio de Janeiro, a prevalência de insegurança alimentar era similar: $28,3 \%$; e a insegurança grave foi observada em 3,7\% dos domicílios.

A prevalência geral de insegurança alimentar em Duque de Caxias (53,8\% ) foi similar àquela observada no Nordeste brasileiro, e a prevalência de insegurança grave $(6,3 \%)$ foi comparável à média nacional. Contudo, esses números representam quase o dobro das médias estimadas na PNAD para o estado do Rio de Janeiro.

Resultados similares aos de Duque de Caxias foram observados em Campinas, no estado de São Paulo. Em estudo de base populacional, em famílias com idosos, a prevalência de insegurança alimentar era de $52,0 \%$ e a de insegurança alimentar grave, de 7,0\% ${ }^{17}$.

Na população norte-americana, inquérito de base domiciliar, realizado em 2005, revelou que $11,0 \%$ da população encontravam-se em situação de insegurança alimentar ${ }^{23}, 0$ que é bem menor do que foi observado neste estudo. Entretanto, a prevalência de segurança alimentar muito baixa entre as famílias da América do Norte $(3,9 \%)^{24}$ apresentou-se semelhante à média brasileira de insegurança alimentar grave, e comparável àquela observada entre as famílias de Duque de Caxias que recebiam renda mensal entre meio e um salário-mínimo per capita.

A prevalência significativa de insegurança alimentar observada entre as famílias de Duque de Caxias, possivelmente, é resultado das condições de vida precárias a que está submetida a população dessa área. Em Duque de Caxias, $73,0 \%$ das famílias tinham renda menor que um salário-mínimo per capita. Assinale-se que somente acima desse limite não foram observadas famílias com insegurança alimentar grave, embora $5,0 \%$ fossem considerados na categoria insegurança alimentar moderada; diferentemente da $\mathrm{PNAD}^{7}$, quando foi observada insegurança alimentar moderada/grave até entre famílias que recebiam mais de três salários-mínimos per capita por mês $(1,0 \%)$.

Entre as famílias de Duque de Caxias que recebiam mensalmente menos de $1 / 4$ do saláriomínimo per capita, apenas 12,0\% relataram segurança alimentar. No Brasil, em 2004, entre as famílias com essa faixa de renda, $17,5 \%$ se consideravam em segurança alimentar e na Região Sudeste, $23,8 \%{ }^{7}$. Nessa categoria de renda, as prevalências de insegurança alimentar moderada $(25,2 \%$ ) e grave $(21,3 \%)$ foram elevadas; porém, esses dad os são discretamente mais favoráveis do que aqueles observados para o País: na PNAD, $61,2 \%$ dos domicílios com renda até esse limite foram categorizados em insegurança alimentar moderada ou grave ${ }^{7}$. Os dados de Caxias foram comparáveis aos observados para a Região Sudeste, onde 50,8\% dos domicílios que auferiam renda per capita até $1 / 4$ do salário-mínimo foram considerados em insegurança alimentar moderada ou grave ${ }^{7}$.

Neste estudo, a escolaridade do chefe da família associou-se significantemente à insegurança alimentar; observou-se que a prevalência de segurança alimentar foi crescente conforme aumentou o grau de escolaridade do chefe da família. Em contrapartida, a proporção de famílias em insegurança alimentar moderada e grave reduziu-se com o incremento da escolaridade do chefe da família; embora a situação de insegurança alimentar leve não variasse de maneira linear com a escolaridade do chefe da família. 
Nnakwe \& Yegammia ${ }^{25}$ observaram que a situação de segurança alimentar em famílias de Coimbatore, na Índia, estava associada significantemente com o grau de escolaridade do chefe da família.

A categorização das famílias segundo os critérios da ABIPEME também mostrou capacidade de discriminação para segurança alimentar: nas categorias socioeconômicas mais privilegiadas (classes A e B) não foram observadas famílias em insegurança alimentar grave e a prevalência da insegurança alimentar moderada foi desprezível. Enquanto que nas classes mais desfavorecidas ( $D$ e E), aproximadamente, $70 \%$ das famílias relataram algum grau de insegurança alimentar.

Em Duque de Caxias, a segurança alimentar mostrou-se significantemente associada ao número de moradores no domicílio. Observou-se incremento significativo na prevalência de insegurança alimentar grave à medida que o número de moradores aumentou. Entre as famílias com pelo menos oito moradores, $48,0 \%$ das famílias foram classificadas em situação de insegurança alimentar moderada ou grave e a prevalência de insegurança grave superou a de segurança alimentar. Estes dados são similares aos da PNAD para o Brasil, que revelaram que $42,6 \%$ das famílias com sete ou mais moradores apresentaram segurança alimentar moderada ou grave.

A ausência de associação entre as condições de saneamento básico e segurança alimentar relaciona-se com as condições gerais de abastecimento de água e esgoto sanitário na região de estudo, que não permitem discriminação entre as famílias. Por outro lado, a presença de filtro para o tratamento de água para o consumo apresentou-se significantemente associada à segurança alimentar. Esta variável pode estar indicando melhores condições de acesso a bens e serviços e/ou mais preocupação com a saúde.

As razões de prevalências, comparando as categorias extremas das variáveis socioeconômicas e de saneamento, que se mostraram associadas à segurança alimentar permitem identificar os indicadores que apresentariam maior capacidade de discriminação entre as categorias de segurança alimentar e de insegurança grave. A renda familiar mensal per capita em salários-mínimos, categorizada nos pontos de corte que são utilizados pelo $\mathrm{IBGE}^{7}$, mostrou ser um indicador que discrimina tanto a segurança quanto a insegurança alimentar: famílias com renda per capita mensal abaixo de $1 / 4$ do salário-mínimo têm 21 vezes mais chance de apresentar insegurança do que aquelas com renda acima de um salário-mínimo. Esse indicador sugere que, aproximadamente, os 14\% das famílias do distrito de Campos Elíseos (Duque de Caxias, RJ) que em 2005 auferiam renda inferior a esse limite deveriam ser incluídos em programas de transferência de renda. Entretanto, nesta pesquisa, somente $9 \%$ (intervalo de confiança de 95\% : 7\%-12\% ) das famílias de Campos Elíseos foram identificadas como beneficiárias de programas de transferência de renda oficiais.

Os dados apresentados evidenciam a dimensão da insegurança alimentar frente às condições socioeconômicas da população investigada. 0 município de Duque de Caxias representa pólo importante de geração de renda, concentra grande número de indústrias e de estabelecimentos comerciais, possui o sexto maior Produto Interno Bruto entre os municípios brasileiros e é o segundo município com maior arrecadação do estado do Rio de Janeiro ${ }^{26}$. No próprio distrito estudado localiza-se a segunda maior refinaria de petróleo do País. Entretanto, os dados apresentados revelam que a situação social e econômica da população dessa região é incoerente com a riqueza gerada no município. A desigualdade na distribuição de renda e no acesso a bens e serviços, resultante da exclusão social, compromete as condições de alimentação. Ações de responsabilidade social que visem à garantia da segurança alimentar são necessárias nessa área, tanto por parte do poder público como da iniciativa privada. 
A GRADECIMENTOS

À colaboração de Luiz Alberto M atzembacher (IBGE), pela elaboração do banco de dados e pelo apoio logístico na etapa de digitação dos questionários.

\section{COLABORADORES}

R. SALLES-COSTA foi responsável pelo desenho do estudo, pela coordenação da investigação de campo, pelo desenho, pela análise e interpretação de dados e pela redação do artigo. R.A. PEREIRA foi responsável pelo desenho do estudo e pela redação do artigo. M.T.L. VASCONCELLOS foi responsável pelo desenho do estudo, pelo plano amostral e pelo plano de análises expandidas. G.V. VEIGA foi responsável pelo desenho do estudo e pela redação do artigo. V.M.R. MARINS foi responsável pelo desenho do estudo e pela redação do artigo. B.C. JARDIM foi responsável pelo desenho do estudo. F.S. GOMES foi responsável pelo desenho do estudo, pela análise e interpretação de dados e pela redação do artigo. R. SICHIERI idealizou a proposta da pesquisa, foi responsável pelo financiamento, participou de todas as etapas de elaboração do estudo, da análise e da interpretação de dados e da redação do artigo.

\section{RE FERÊ N C IAS}

1. Radimer KL, Olson CM, Greene JC, Campbel CC, Habitch J-P. Understanding hunger and developing indicators to assess it in women and children. J Nutr Educ. 1992; 24(1Suppl):36S-45S.

2. Álvarez MC, Estrada A, Montoya EC, MelgarQuiñónez H. Validación de escala de la seguridad alimentaria doméstica en Antioquia, Colombia. Salud Publica Mex. 2006; 48(6):474-81.

3. Melgar-Quinonez $H$, Zubieta AC, Valdez E, Whitelaw B, Kaiser L. Validation of an instrument to monitor food insecurity in Sierra de Manantlan, Jalisco. Salud Publica Mex. 2005; 47(6):413-22.

4. Melgar-Quinonez HR, Zubieta AC, McNelly B, Nteziyaremye A, Gerardo MF, Dunford C. Household food insecurity and food expenditure in Bolivia, Burkina Faso, and the Philippines. J Nutr. 2006; 136(5):1431S-7S.

5. Pérez-Escamilla RS, Randolph I, Hathie IG. Adaptation and validation of the USDA food security scale in rural Senegal [Abstract \# 104.1] Faseb J. 2004; 18(Suppl):A106.

6. Perez-Escamilla R, Segall-Correa AM, Maranha LK, Sampaio MFA, Marin-Leon L, Panigassi G. An adapted version of the U.S. Department of Agriculture Food Insecurity module is a valid tool for assessing household food insecurity in Campinas, Brazil. J Nutr. 2004; 134(8):1923-8.

7. Instituto Brasileiro de Geografia e Estatística. Pesquisa nacional por amostra de domicílios (PNAD) 2004: segurança alimentar. Rio de Janeiro: IBGE; 2006. p.148.

8. Freitas MCS. Segurança alimentar e nutricional: algumas considerações 2006 [acesso em 2007 jun 13]. Disponível em: <http://www.comciencia.br/ reportagens/2005/09/10.shtml>.

9. Habicht JP, Pelto G, Frongillo E, Rose D. Conceptualization and instrumentation of food insecurity. National Academy of Sciences Workshop [cited 2007 Jun 13]. Available from: <http://w w w 7. nationalacademies.org/cnstat/ Conceptualization_and_Instrumentation_of_ Food_Security_Paper.pdf $>$.

10. Campbell CC. Food insecurity: a nutritional outcome or a predictor variable? J Nutr. 1991; 121: 408-15,

11. Costa-Neto C. Vila Rosário: o resgate de uma sociedade pela ciência, pela tecnologia, pelo trabalho e pela compreensão. Rio de Janeiro: Cálamo Produção Editorial; 2002. p.476.

12. Monteiro CA. A dimensão da pobreza, da desnutrição e da fome no Brasil: implicações para políticas públicas. Instituto Nacional de Altos Estudos. Seminário Especial Fome e Pobreza. 2003 [acesso 2006 dez 10]. Disponível em: <http:// forumnacional.org.br>.

13. Rocha S, Albuquerque RC. Geografia da pobreza extrema e vulnerabilidade à fome. Seminário Especial Fome e Pobreza. Estudos e Pesquisas, no 54. Rio de Janeiro; 2003.

14. Instituto Brasileiro de Geografia e Estatística. Censo Demográfico 2000. Rio de Janeiro.

15. Census and survey processing system version 3.2. international programs center. Washington (DC): Census Bureau; 2005.

16. Segall-Corrêa AM, Pérez-Escamilla R, Maranha LK, Sampaio MFA, Marin-León L, Panigassi G, et al. Projeto: acompanhamento e avaliação da segurança alimentar de famílias brasileiras: validação de metodologia e de instrumento de coleta de informação. Campinas: Unicamp; 2003. Relatório Técnico.

17. Marín-León L, Segall-Corrêa AM, Panigassi G, Maranha LK, Sampaio MFA, Pérez-Escamilla R. A 
percepção de insegurança alimentar em famílias com idosos em Campinas, São Paulo, Brasil. Cad Saúde Pública. 2005; 21(5):1433-40.

18. Mattar FN. Análise crítica dos estudos de estratificação sócio-econômica de ABA. ABIPEME Rev Admin. 1995; 30(1):57-74.

19. Rao JNK, Scott AJ. On chi-squared tests for multiway contingency tables with cell proportions estimated from survey data. Ann Statist. 1984; $12(1): 46-60$.

20. $R$ [computer program]. Version 2.5.0. The $R$ Foundation for Statistical Computing; 2007. Viena; 2007.

21. Wolter KM. Introduction to variance estimation. New York: Springer; 2003.

22. STATA [computer program]. Version 9.0. College Station (TX): Stata Corporation; 2006.

23. Nord M, Andrews M, Carlson S. Household food security in the United States, 2005. Washington
(DC): United States Department of Agriculture; 2006. Food Assistance and Nutrition Research Report, 29 [cited 2007 Jun 13]. Available from: <http://ww w.ers.usda.gov/Publications/err29/>.

24. Bickel G, Nord M, Price C, Hamilton W, Cook J. $M$ easuring food security in the United States: guide to measuring household food security. Alexandria: U.S. Department of Agriculture; 2000.

25. Nnakwe N, Yegammia C. Prevalence of food insecurity among households with children in Coimbatore, India. Nutr Res. 2002; 22(9): 1009-16.

26. Instituto Brasileiro de Geografia e Estatística. Produto interno bruto dos municípios. [acesso 2005 abr 24]. Disponível em: <http://www.ibge. gov.br>.

Recebido em: 19/6/2007

Versão final reapresentada em: 16/10/2007

Aprovado em: 31/3/2008 
